the programme. Thus, to bridge the gaps, in 2015 the Ministry of Public Health adopts the "Merit Maker for Child Drowning Prevention" measure, aiming to identify the networks working on drowning prevention that covers at least six key measures, emphasising community-based multi-disciplinary approach, resource sharing, participation of local agencies, and rewarding Merit Makers whose contributions are in accordance with the established criteria.

Conclusion Since the launch of the programme on child drowning prevention, policies and measures have been implemented, resulting in a constant reduction in child drowning fatalities. In such efforts, the Ministry of Public Health has also revised several measures so that they are more effective in the country in achieving the child drowning reduction goal.

\section{SURVIVAL SWIMMING - EFFECTIVENESS OF SWIMSAFE IN PREVENTING DROWNING IN MID AND LATE CHILDHOOD}

${ }^{1,2}$ Abu Talab, ${ }^{1,2}$ Aminur Rahman, 1,2 Fazlur Rahman, ${ }^{1,2}$ Jahangir Hossain, ${ }^{3}$ Justin Scarr, ${ }^{4}$ Michael Linnan. ${ }^{1}$ International Drowning Research Centre - Bangladesh (IDRCB); ${ }^{2}$ Centre for Injury Prevention and Research, Bangladesh (CIPRB); ${ }^{3}$ Royal Life Saving Society Australia; ${ }^{4}$ the Alliance for Safe Children (TA SC)

\subsection{6/injuryprev-2016-042156.270}

Introduction Conclusive evidence of the protective effect of swimming has been lacking in high income countries. Nested case control studies in surveys done across Asia have shown significant associations between swimming ability and protection against drowning in children four years and older. In order to confirm that the relationship between swimming ability and protection from drowning was a causal relationship, a four year cohort study of a structured survival swimming program known as SwimSafe was undertaken in Bangladesh.

Methods A cohort study was conducted to compare the protection from drowning conferred by participation in the SwimSafe survival swimming. The survival swimming cohort was made up of children primarily aged 4-12 years old who were graduates of the SwimSafe program in rural Bangladesh. The non-survival swimming cohort was made up of age- and sex-matched children from neighbouring villages who were not participants in the SwimSafe program. Death from drowning was compared in both groups using survival analysis techniques.

Results A total of 81,659 children had been taught survival swimming in the study area who had been followed through September 30, 2010 and whose survival status was known. A cohort of 140,479 non-SwimSafe participants had been followed in the same system and whose survival status was known. Children from this non-SwimSafe cohort were matched on age and sex with SwimSafe graduates and a total of 66,066 children from each cohort were fully matched. The matching cohorts were subjected to Kaplan Meier and Cox Proportional Hazard survival analyses with fatal drowning as the primary outcome. These showed a substantial and statistically significant reduction in relative risk of drowning in SwimSafe children as compared to the matched non-SwimSafe children.

Conclusions Survival swimming, as taught in the SwimSafe survival swimming program with its highly structured and skills based methodology confers protection from drowning in children who graduate from the SwimSafe program.

\section{UNINTENTIONAL DROWNING AND PSYCHOACTIVE SUBSTANCES}

${ }^{1}$ Tuulia Pajunen, ${ }^{1}$ Philippe Lunetta, ${ }^{2}$ Gordon Smith, ${ }^{3}$ Erkki Vuori, ${ }^{4}$ Pirjo Lillsunde, ${ }^{5}$ Frank F Vincenzi. ' University of Turku, Finland; ${ }^{2}$ University of Maryland, USA; ${ }^{3}$ University of Helsinki, Finland; ${ }^{4}$ National Institute for Health and Welfare, Finland; ${ }^{5}$ University of Washington, USA

\subsection{6/injuryprev-2016-042156.271}

Background Alcohol is a well-known risk factor in unintentional drownings. While psychotropic drugs may also cause psychomotor impairment and affect cognition, no detailed studies have focused on their association with drowning. Finland provides extensive post-mortem toxicological data for studies on drowning due to its high medico-legal autopsy rates.

Methods Drowning cases, 2000 through 2009, for which postmortem toxicological analysis was performed, were selected from the database of Toxicological laboratory, Department of Forensic Medicine, University of Helsinki, using the ICD-10 nature of injury code T75.1. The data were narrowed down to unintentional drowning, using the ICD-10 external injury codes V90, V92 and W65-74. For each drowning case, the blood alcohol concentration (BAC) and the concentrations of other drugs were recorded. The contribution of psychotropic drugs to drowning was evaluated based on their blood concentration by mean of a 6-grade scale.

Results Among victims $\geq 15$ years old, there were 1697 unintentional drownings. $303(17.8 \%)$ were boating-related and 1394 $(82.2 \%)$ non-boating-related. $65.0 \%$ of boating-related and $61.8 \%$ of non-boating-related victims were alcohol-positive $(=\mathrm{BAC} \geq 0.5 \%$ ) $)$. The male to female ratio in alcohol-positive drownings was 7.3. At least one psychotropic drug was found in $453(26.7 \%)$ drowning cases, with some victims having up to 7 different drugs. Overall 70 different psychotropic drugs were detected. 134 (7.9\%) cases were both alcohol-negative and psychotropic drug -positive, of them 59 (3.5\%) were graded 4 to 6 , indicating possible to very probable contribution to drowning. Conclusions Psychotropic drugs alone or in association with alcohol may be an overlooked risk factor to drowning due to their effects on psychomotor function and cognition. Future studies should also address other mechanisms, for instance druginduced long QT-syndrome, by which drugs may contribute to drowning.

\section{EVALUATION OF MEASURES FOR THE MANAGEMENT OF HIGH-RISK WATER SOURCES IN COMMUNITIES FOR CHILD DROWNING PREVENTION IN THAILAND}

Suchada Gerdmongkolgan, Som Ekchaloemkiet. Department of Diseases Control, Ministry of Public Health, Thailand

\subsection{6/injuryprev-2016-042156.272}

Background In Thailand, drowning is the number one cause of death among children under 15 years of age, whose drowning death rate ranged from 7.6 to 11.5 per 100,000 child population between 2004 and 2013 .

Objective To evaluate the measures for the management of highrisk water sources in communities by installing warning signboards and easily available water safety devices for child drowning prevention.

Methods This evaluative research was conducted in two types of communities: one with the installation of warning signboards on water safety and advice for assisting drowning victims and 